\title{
Is Weight-Bearing Asymmetry Associated with Postural Instability after Stroke? A Systematic Review
}

\author{
Jip F. Kamphuis, ${ }^{1}$ Digna de Kam, ${ }^{2}$ Alexander C. H. Geurts, ${ }^{2,3}$ and Vivian Weerdesteyn ${ }^{2,3}$ \\ ${ }^{1}$ ViaReva, Centre for Rehabilitation, P.O. Box 812, 7301 BB Apeldoorn, The Netherlands \\ ${ }^{2}$ Radboud University Medical Centre, Nijmegen Centre for Evidence Based Practice, \\ Department of Rehabilitation, P.O. Box 9101, 6500 HB Nijmegen, The Netherlands \\ ${ }^{3}$ Sint Maartenskliniek, Research, Development \& Education, P.O. Box 9011, 6500 GM Nijmegen, The Netherlands
}

Correspondence should be addressed to Vivian Weerdesteyn; v.weerdesteyn@reval.umcn.nl

Received 31 October 2012; Revised 28 January 2013; Accepted 18 March 2013

Academic Editor: Catherine Said

Copyright (C) 2013 Jip F. Kamphuis et al. This is an open access article distributed under the Creative Commons Attribution License, which permits unrestricted use, distribution, and reproduction in any medium, provided the original work is properly cited.

Introduction. Improvement of postural stability is an important goal during poststroke rehabilitation. Since weight-bearing asymmetry (WBA) towards the nonparetic leg is common, training of weight-bearing symmetry has been a major focus in poststroke balance rehabilitation. It is assumed that restoration of a more symmetrical weight distribution is associated with improved postural stability. Objective. To determine to what extent WBA is associated with postural instability in people after stroke. Methods. Electronic databases were searched (Cochrane, MEDLINE, EMBASE, and CINAHL) until March 2012. Main Eligibility Criteria. (1) Participants were people after stroke. (2) The association between WBA and postural stability was reported. Quality of reporting was assessed with the STROBE checklist and a related tool for reporting of confounding. Results. Nine observational studies met all criteria. Greater spontaneous WBA was associated with higher center of pressure (COP) velocity and with poorer synchronization of COP trajectories between the legs (two and one studies, resp.). Evidence for associations between WBA and performance on clinical balance tests or falls was weak. Conclusion. Greater WBA after stroke was associated with increased postural sway, but the current literature does not provide evidence for a causal relationship. Further studies should investigate whether reducing WBA would improve postural stability.

\section{Introduction}

Of all possible sensorimotor consequences of stroke, impaired postural stability probably has the greatest impact on gait and independency in activities of daily living (ADL) $[1,2]$. Indeed, to achieve improvement in walking capacity or ADL, improvement in standing balance is more important than improvement in leg strength [3]. Moreover, balance impairments are a major risk factor for falls [4]. Falling is a very common complication after stroke, with as many as $50 \%$ to $70 \%$ of the people who return home from the hospital or rehabilitation center experiencing falls [5]. These falls can have severe consequences such as hip fractures and decreased physical activity due to fear of falling [4]. Improvement of postural stability is, therefore, an important goal in stroke rehabilitation $[2,3]$.

Another common consequence of stroke is weightbearing asymmetry (WBA). During quiet stance a substantial amount of WBA in favor of the nonparetic leg is commonly observed [6-8]. Although asymmetry significantly improves during the first weeks of rehabilitation, some degree of WBA persists (on average 10\% more weight being borne on the nonparetic leg) [9-11]. This asymmetry increases during dualtask performance [11], suggesting that weight-bearing on the paretic leg is not easily performed automatically.

Historically, training of weight-bearing symmetry has been a major focus in balance rehabilitation after stroke. Facilitation of normal movement patterns and symmetry in weight-bearing are among the key principles of the Bobath concept and related Neurodevelopmental Treatment (NDT) [12]. These training approaches implicitly assume that restoration of a more symmetrical weight distribution is associated with improved postural stability [13]. In the same vein, several specific interventions, such as compelled weightbearing through shoe lifts and biofeedback training, have been developed to re-establish weight-bearing symmetry in 
people after stroke $[6,14-17]$. Weight-bearing asymmetry was reduced by these interventions; however, whether this was associated with improvement of postural control was not reported.

In order to better understand the clinical meaning of weight-bearing asymmetry for balance rehabilitation after stroke, it is important to know how WBA and postural stability are correlated. The aim of this systematic review was, therefore, to determine to what extent WBA is associated with postural instability in people after stroke. For this review a broad definition of postural stability was chosen: "the ability of a person to maintain, achieve or restore balance, or to avoid falling" [18].

\section{Methods}

A systematic review was conducted according to the PRISMA statement where applicable [19]. It should be noted that the PRISMA statement was designed for systematic reviews of intervention studies and, therefore, several points could not be addressed in this study.

2.1. Eligibility Criteria. To be included studies had to meet the following eligibility criteria:

(1) publication type: there were no restrictions on type of study; however, only papers published in peerreviewed journals were included;

(2) participants: subjects had to have sustained a stroke, regardless of stroke type or poststroke duration;

(3) outcome measures: the study contained data on the association between WBA and (any measure of) postural stability. Intervention studies aimed at restoring weight-bearing symmetry were only considered for inclusion if they reported on the association between WBA and postural stability. Measures for WBA were accepted if they quantified the differences in weight borne on either leg;

(4) language: the study was written in the English, German, or Dutch language.

2.2. Information Sources. Studies were identified by searching the following electronic databases: Cochrane, MEDLINE, EMBASE, and CINAHL. The search was conducted by the first author (J. Kamphuis) in March 2012. There were no restrictions regarding publication date. In addition, reference lists of the included studies were screened for potentially eligible studies.

2.3. Search Strategy. The following MESH terms were used for the search in MEDLINE:

(1) "Stroke," "Brain Infarction", "Cerebrovascular Disorders", "Brain Ischemia”, "Paresis”, "Hemiplegia”, "Intracranial Hemorrhages", "Intracranial Embolism and Thrombosis"

(2) "Weight-Bearing"

(3) "Postural Balance", "Posture"
Details on the full search strategy in MEDLINE are available in Appendix A. For the other databases the same terms were used where possible.

2.4. Study Selection. Study selection was initially performed by the first author (J. Kamphuis) and then checked by the second author (D. de Kam). First, the titles and abstracts of the publications retrieved by electronic searching were screened. Second, potentially eligible studies were retrieved full text before definitive inclusion. In the case of disagreement between the two authors, the last author (V. Weerdesteyn) was consulted to decide whether a study was included.

2.5. Data Extraction. Data were extracted from the studies by J. Kamphuis and then checked by D. de Kam. The extracted data were discussed until consensus was reached. In the case of disagreement the last author (V. Weerdesteyn) was consulted. No specific form was used for data extraction; however, the outcomes to be extracted were defined a priori. The following information was extracted from each study:

(1) author and year of publication,

(2) study design,

(3) number of participants,

(4) demographic participant characteristics: age and time after stroke,

(5) baseline measures of stroke severity,

(6) baseline measures of clinical balance and/or ambulatory performance,

(7) dependent measures of postural stability,

(8) statistics for association between WBA and postural stability.

2.6. Definition of Measures of Postural Stability. Of each study we summarized the reported results regarding the association between postural stability and WBA, regardless whether this association was the focus of the original study or not (Table 1). Because a variety of measures of postural stability have been reported in the literature, they were categorized by the methods of data collection:

(1) quiet standing posturography: postural sway measured with force plates, usually expressed as center of pressure (COP) amplitude or COP velocity,

(2) dynamic posturography: postural responses to balance perturbations. These responses are measured quantitatively in terms of COP excursions, muscle onset latencies, kinematic parameters etcetera;

(3) clinical balance tests: these tests are typically used by clinicians to evaluate balance performance during functional tasks such as reaching or one-legged standing;

(4) falls in daily life: the ultimate consequence of postural instability is falling. Therefore, the number of falls in daily life was also considered as a measure of postural stability. 


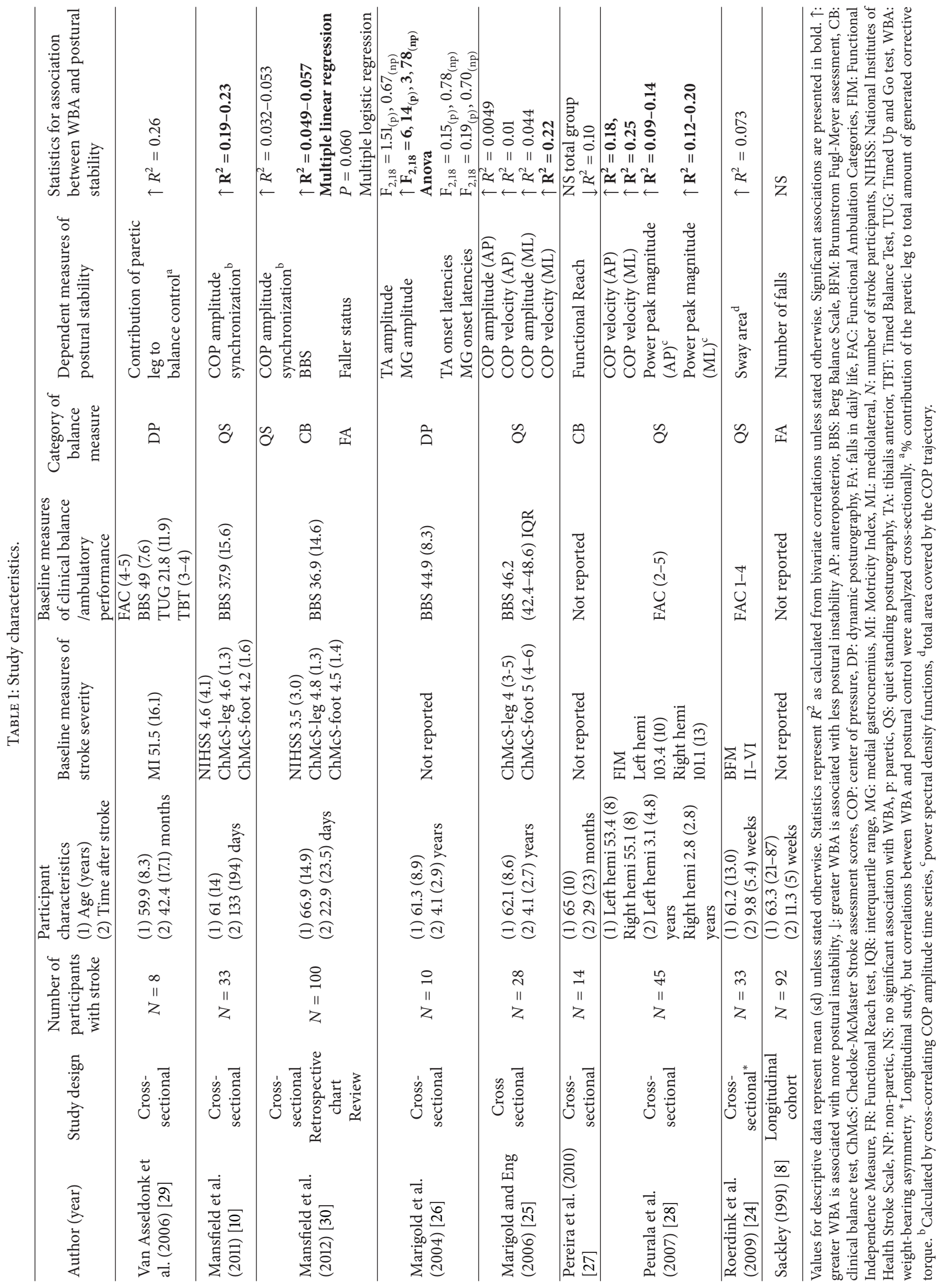


Results regarding the association between postural stability and WBA were presented as

(1) positive: if greater WBA was associated with more postural instability,

(2) negative: if greater WBA was associated with less postural instability.

2.7. Quality of Reporting in Individual Studies. In a recent systematic review it was concluded that there is currently no quality assessment tool available for observational studies [20]. We, therefore, used a checklist for authors of observational studies that was developed by a group of expert methodologists, researchers, and editors: "Strengthening the Reporting of Observational Studies in Epidemiology" (STROBE) [21] to globally assess the reporting quality of the included studies. Although this checklist was not intended as a quality assessment tool, the items were considered relevant by a group of experts in the field. Scoring definitions were derived from the STROBE "Explanation and Elaboration" document [22] and are available in Table 4.

The risk of bias of the included studies was assessed with an instrument developed by Groenwold and coworkers [23]. This tool was specifically designed to rate the report of confounding bias in observational studies based on the STROBE statement.

All included studies were scored by J. Kamphuis and D. de Kam independently. Discrepancies were discussed between the two authors until consensus was reached. If necessary, a third assessor (V. Weerdesteyn) was consulted.

\section{Results}

3.1. Study Selection. A total of 247 articles were retrieved by electronic searching. After screening of titles and abstracts 14 articles were selected. Finally, after full text reading of these 14 articles, nine studies met the eligibility criteria (Figure 1).

3.2. Study Characteristics. Characteristics of the nine included studies are reported in Table 1 . Seven were cross-sectional studies and two were longitudinal cohort studies. However, one longitudinal study analyzed the association between WBA and postural stability cross-sectionally and was, therefore, regarded as a cross-sectional study [24]. Seven of the nine included papers were originally designed to determine the association between WBA and a measure of postural stability $[8,10,25-29]$. In the other two studies this association was reported as a secondary analysis or it could be extracted from a larger regression model $[24,30]$.

Descriptive data on balance and gait capacities of the subjects was provided in all studies except two $[8,27]$. These functional capacities varied greatly between the studies, from a Berg Balance Scale (BBS) of 4 points [10] at the lower end, up to a maximum BBS score of $56[10,29,30]$ or being able to walk independently without supervision (Functional Ambulation Categories 4-5) [24, 28, 29]. As for measures of postural stability, quiet standing posturography was done in five studies $[10,24,25,28,30]$, dynamic posturography in two

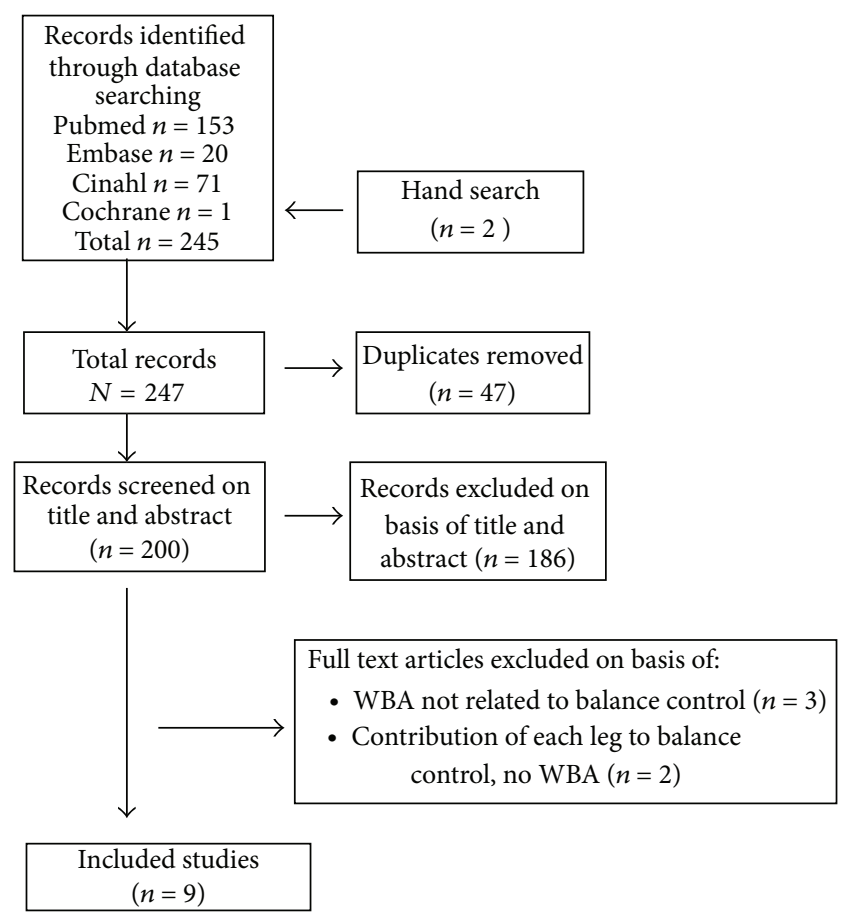

FIGURE 1: Flow chart of systematic review of weight-bearing asymmetry (WBA) related to postural control.

studies [26, 29], clinical balance tests in two studies [27, 30], and number of falls was recorded in two studies $[8,30]$. The study by Mansfield et al. [30] assessed postural control using all categories except dynamic posturography.

3.3. Quality of Reporting in Individual Studies. The quality of reporting of individual studies according to the STROBE criteria and the scores on the risk-of-bias checklist are shown in Tables 2 and 3.

The criteria for the reporting of study rationale, objectives, outcome variables, data analysis, and study results were satisfied in at least seven of the nine studies. Criteria for reporting recruitment sites and methods of participant selection were only sufficiently reported in five and six studies, respectively. In addition, none of the studies clearly justified their sample size. The generalizability of the results was discussed in only five of the nine studies.

For the reporting of confounding bias only one study [30] scored seven points (out of 8 ). The remaining studies had a total score of four points or less (Table 3 ). Three studies applied a method to correct for potential confounders in their analysis [24, 26, 30]; however, only two studies justified their choice for the selection of potential confounders $[25,30]$. The possibility of unobserved confounding was discussed in five of the studies $[8,25,27,29,30]$.

3.4. Results of Individual Studies. Measures of postural stability varied considerably among the studies, even within each of the four categories. Therefore, meta-analysis of the data was not considered appropriate. 


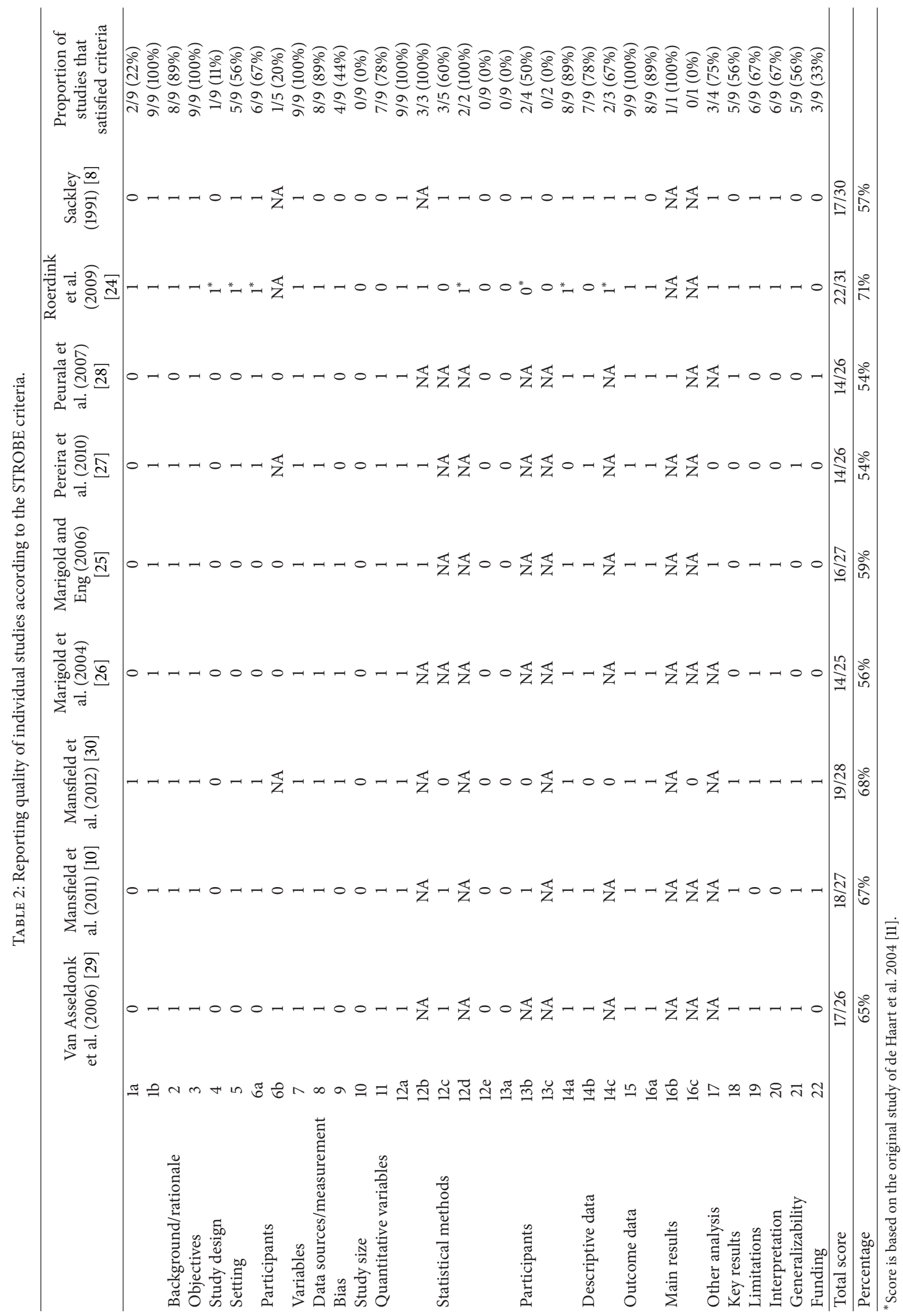


TABLE 3: Reporting of confounding bias in individual studies [23].

\begin{tabular}{|c|c|c|c|c|c|c|c|c|c|c|}
\hline & $\begin{array}{c}\text { Van Asseldonk } \\
\text { et al. (2006) } \\
{[29]}\end{array}$ & $\begin{array}{l}\text { Mansfield } \\
\text { et al. } \\
(2011)[10]\end{array}$ & $\begin{array}{l}\text { Mansfield } \\
\text { et al. } \\
(2012) \\
{[30]}\end{array}$ & $\begin{array}{l}\text { Marigold } \\
\text { et al. } \\
(2004) \\
{[26]}\end{array}$ & $\begin{array}{c}\text { Marigold } \\
\text { and Eng } \\
(2006) \\
{[25]}\end{array}$ & $\begin{array}{l}\text { Pereira } \\
\text { et al. } \\
(2010) \\
{[27]} \\
\end{array}$ & $\begin{array}{c}\text { Peurala } \\
\text { et al. } \\
(2007) \\
{[28]}\end{array}$ & $\begin{array}{l}\text { Roerdink } \\
\text { et al. } \\
(2009) \\
{[24]} \\
\end{array}$ & $\begin{array}{c}\text { Sackley } \\
(1991) \\
{[8]}\end{array}$ & $\begin{array}{l}\text { Number of } \\
\text { studies } \\
\text { satisfied } \\
\text { criteria } \\
\end{array}$ \\
\hline $\begin{array}{l}\text { (1) Reporting of reasons } \\
\text { why potential } \\
\text { confounders are selected } \\
\text { for analysis }\end{array}$ & 0 & 0 & 1 & 0 & 1 & 0 & 0 & 0 & 0 & 2 \\
\hline $\begin{array}{l}\text { (2) Reporting of reasons } \\
\text { to include confounders } \\
\text { in final model }\end{array}$ & 0 & 0 & 1 & 0 & 1 & 0 & 0 & 0 & 0 & 2 \\
\hline $\begin{array}{l}\text { (3) Reporting of } \\
\text { characteristics of key } \\
\text { confounders }\end{array}$ & 0 & 0 & 1 & 0 & 0 & 0 & 0 & 0 & 0 & 1 \\
\hline $\begin{array}{l}\text { (4) Any method used to } \\
\text { control for confounding }\end{array}$ & 0 & 0 & 1 & 1 & 0 & 0 & 0 & 1 & 0 & 3 \\
\hline $\begin{array}{l}\text { (5) Reporting of both } \\
\text { crude and adjusted effect } \\
\text { estimate }\end{array}$ & 0 & 0 & 1 & 0 & 0 & 0 & 0 & 0 & 0 & 1 \\
\hline $\begin{array}{l}\text { (6) Comment on } \\
\text { likelihood of unobserved } \\
\text { confounding }\end{array}$ & 1 & 0 & 1 & 0 & 1 & 1 & 0 & 0 & 1 & 5 \\
\hline $\begin{array}{l}\text { (7) Reporting of a } \\
\text { qualitative statement on } \\
\text { the direction of the } \\
\text { potential effect of } \\
\text { unobserved } \\
\text { confounding }\end{array}$ & 0 & 0 & 1 & 0 & 1 & 0 & 0 & 0 & 0 & 2 \\
\hline $\begin{array}{l}\text { (8) Sensitivity analysis } \\
\text { used to estimate } \\
\text { potential impact of } \\
\text { unobserved } \\
\text { confounding }\end{array}$ & 0 & 0 & 0 & 0 & 0 & 0 & 0 & 0 & 0 & 0 \\
\hline Total score & 1 & 0 & 7 & 1 & 4 & 1 & 0 & 1 & 1 & \\
\hline
\end{tabular}

3.4.1. Definitions of Weight-Bearing Asymmetry. In most studies WBA was expressed as a function of body weight (BW) $[10,26,28-30]$. Most of the studies also considered the direction of WBA (towards the affected or unaffected side) $[8,10,25-28]$, whereas others only calculated the absolute degree of WBA $[24,30]$. In the remaining studies WBA was calculated as the loading ratio between the affected and unaffected leg [27], the difference between loading on the affected versus unaffected leg divided by $50 \%$ of body weight [25], or the deviation of the COP from the midline between the legs in the frontal plane $[8,24]$. Due to these differences it was not possible to compare absolute effect sizes between different studies.

3.4.2. Spontaneous and Imposed Weight-Bearing Asymmetry. In most studies participants were allowed to self-select their weight distribution (spontaneous WBA). In one study participants were instructed to stand as symmetrically as possible [24]. In the experiment of Marigold et al. (2004), three different conditions of WBA were imposed within the same subjects [26].
3.4.3. Associations between Weight-Bearing Asymmetry and Quiet Standing Posturography. The correlation between spontaneous WBA and quiet standing balance was determined in four studies $[10,25,28,30]$. In another study, the association between imposed weight-bearing symmetry and postural sway was reported [24].

Two cross-sectional studies with a total sample of 73 participants in the chronic phase after stroke analyzed the correlation of WBA with measures of postural sway [25, 28]. Both studies reported that greater WBA was associated with more postural sway. These associations were most evident for COP velocity; however, the explained variance was moderate $\left(R^{2}=0.18-0.25\right)$. The study by Roerdink et al. did not find a significant association between WBA and total sway area [24]. In this study, however, participants were instructed to stand as symmetrically as possible.

In the two studies by Mansfield et al. $[10,30]$ the correlation was examined between WBA and between-limb synchronization of COP trajectories. Synchronization was expressed as the cross-correlation coefficient between the individual COP trajectories under the left and right foot. 


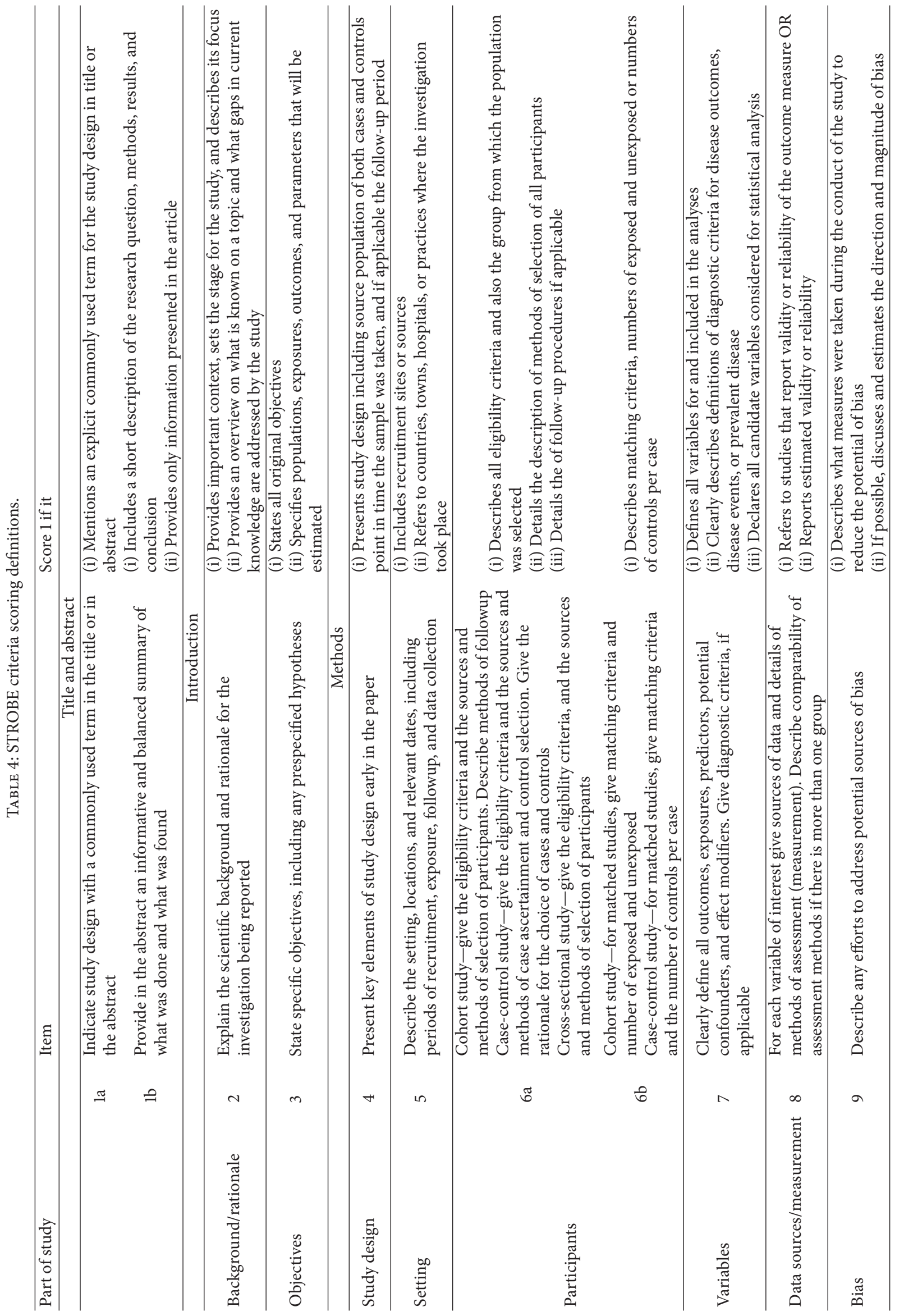




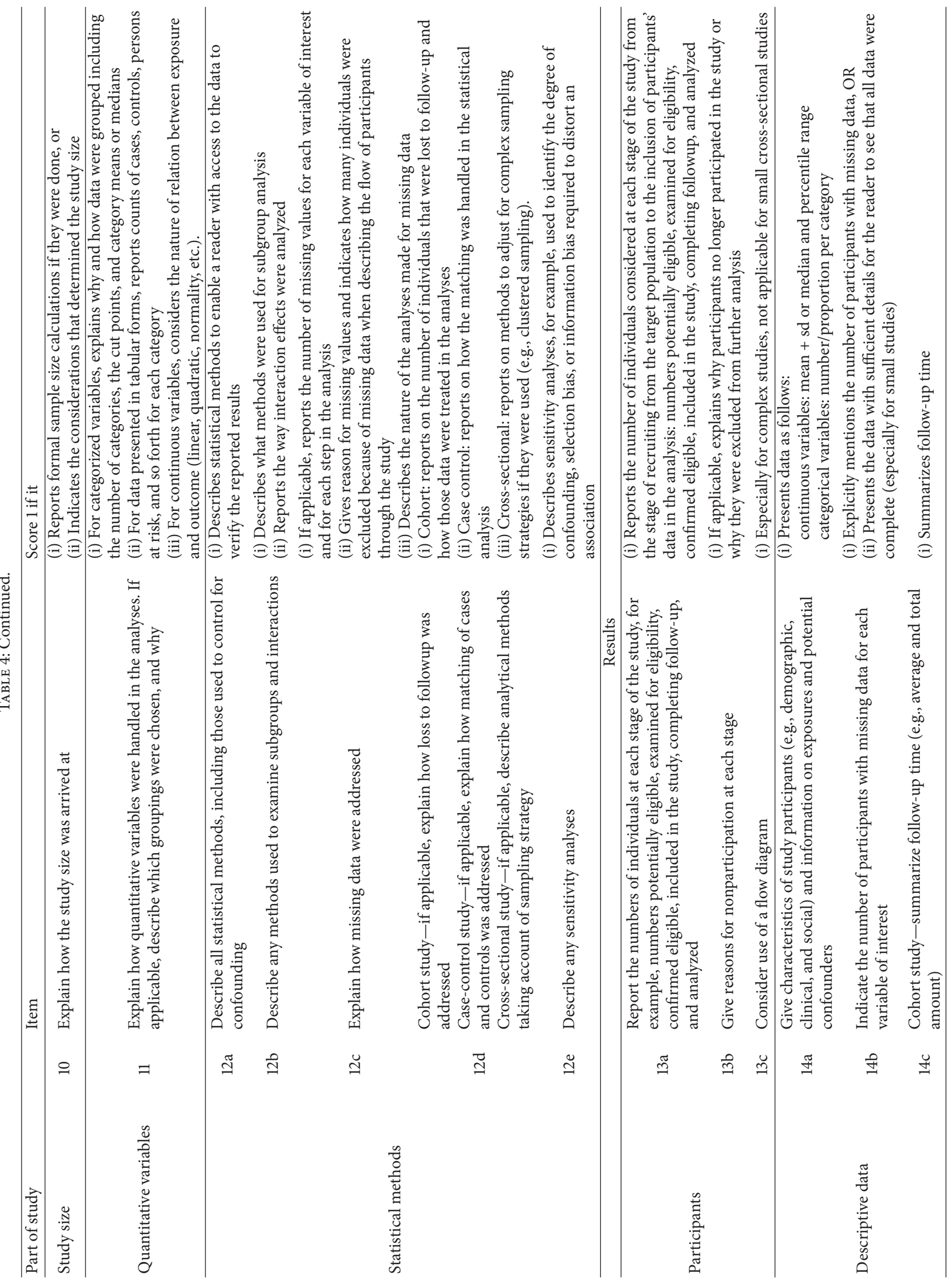




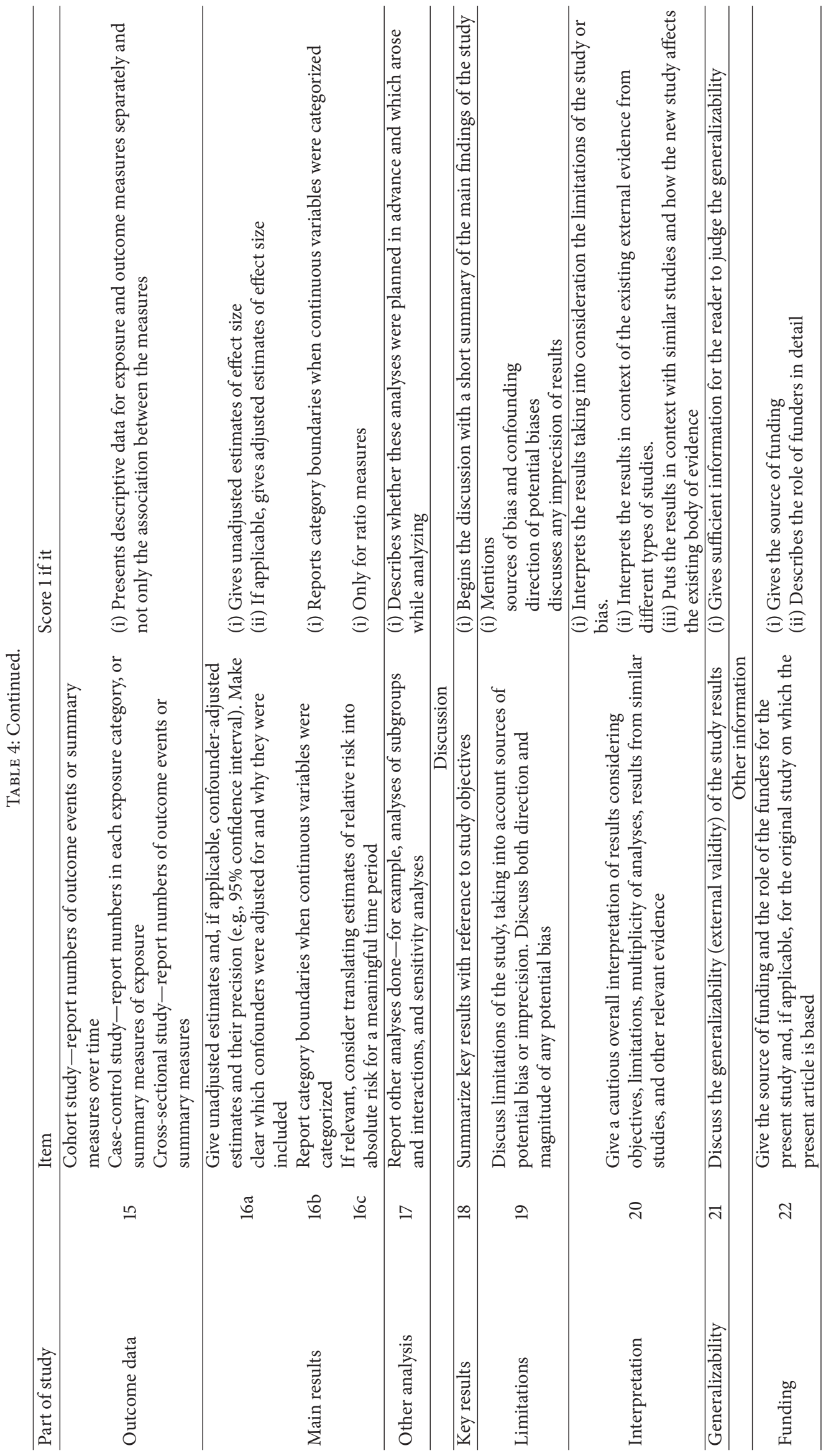


High correlation coefficients indicated that regulatory activity was synchronized between the paretic and nonparetic legs and was interpreted as the two legs working together to control posture. In both studies, WBA was associated with low between-limb synchronization, but this was significant in only one of the studies $\left(R^{2}=0.19-0.23\right)[10]$.

3.4.4. Associations between Weight-Bearing Asymmetry and Dynamic Posturography. The correlation between WBA and dynamic posturography outcomes was investigated in two studies [26, 29]. These studies included a total of 18 individuals with stroke. Participants stood on a platform and were instructed to maintain their balance, while random platform movements (discrete [26] or continuous [29]) were applied in forward and backward directions. The study of Marigold et al. [26] used a within-subjects design with three different stance conditions of imposed WBA: increased weight-bearing load, decreased weight-bearing load, and selfselected stance. This study found no significant differences in muscle onset latency or amplitude of muscle activity between the three load conditions in individuals with stroke, except for an increase in gastrocnemius amplitudes with more weightbearing in both the paretic and nonparetic limbs. In contrast, in healthy controls increased loading shortened the onset latencies of gastrocnemius and increased the amplitude of tibialis anterior and gastrocnemius responses.

In the study by van Asseldonk et al. [29] the "dynamic balance contribution" of each leg to postural control was assessed by comparing the ankle joint torques of the paretic with the nonparetic limb in individuals with stroke and healthy subjects. A key finding of this study was that the contribution of the paretic leg to balance was much smaller (on average about 10-20\%) than its contribution to weight-bearing (on average about $40-45 \%$ ). More weight-bearing on the paretic leg tended to be associated with a larger contribution to postural control; however, this correlation was not significant $\left(R^{2}=0.26, P=0.24\right)$. Conversely, in healthy subjects adopting an asymmetrical posture, the contribution of each leg to postural control equaled its contribution to weightbearing.

3.4.5. Associations between Weight-Bearing Asymmetry and Clinical Balance Performance Tests or Falls in Daily Life. The correlation between WBA and clinical balance performance tests was examined in two studies [27, 30]. Although Mansfield et al. [30] observed a significant association of greater WBA with poorer BBS scores, the explained variance was very low (5-6\%). It must be mentioned, however, that their study was not designed to specifically investigate this relationship and that the reported association was derived from a larger regression model. In the study by Pereira et al. [27] no significant correlations between Functional Reach and WBA were found for the total number of 14 participants. However, when only the patients with WBA towards the nonparetic leg were considered $(n=10)$, greater WBA was significantly associated with better Functional Reach scores $\left(R^{2}=0.49\right)$.

The association between WBA and falls was measured in two studies $[8,30]$. In the study of Sackley [8] falls were recorded both prospectively and retrospectively from patient charts and by interviews. Mansfield and coworkers retrospectively collected data on both falls and postural stability from patient charts [30]. Both studies did not find significant associations. Again, the association reported by Mansfield and coworkers [30] was derived from a regression analysis that was conducted to answer a different research question.

\section{Discussion}

The purpose of this review was to determine the association between WBA and postural stability in people after stroke. Nine observational studies were found, of which seven were originally designed to investigate this relationship. Measures of postural stability were very diverse among the included studies and were, therefore, categorized according to the methods used for data collection. For static posturography the general trend was that greater WBA was associated with larger COP velocities. The strengths of the associations were, however, moderate at best.

4.1. Static and Dynamic Posturography. In two studies that recorded overall COP excursions (i.e., of the paretic and nonparetic leg combined) greater WBA was associated with increased postural sway $[25,28]$. Interestingly, the observed associations appeared to be stronger for measures of COP velocity than $\mathrm{COP}$ amplitude. COP velocity measures are not only more reliable than measures of COP amplitude $[31,32]$, but also sensitive to changes in the frequency of regulation. This is important because particularly the higher frequencies within the COP fluctuations reflect the stabilizing ankle torques. Hence, the finding that greater WBA was more strongly associated with greater COP velocities than COP amplitudes suggests that more regulatory activity was applied for maintaining upright stability when a more asymmetrical weight distribution was adopted.

Several other studies included in this review measured the individual paretic and nonparetic COP trajectories, which enabled the researchers to determine the regulatory activity of each leg separately $[10,24,29,30]$. In healthy people, adopting an asymmetric weight distribution results in increased regulatory activity (i.e., COP velocities) under the most loaded leg, thereby increasing its relative contribution to postural control [33]. In the studies by Roerdink et al. [24] and van Asseldonk et al. [29] the regulatory activity under the paretic leg was found to be substantially lower compared to the nonparetic leg. In the study by van Asseldonk et al. [29] the explained variance $\left(R^{2}=0.26\right)$ between WBA and the contribution of the paretic leg to postural control was in the same order of magnitude as the values reported for overall COP velocity $\left(R^{2}=0.18-0.25\right)$, but the correlation did not reach significance, possibly due to the limited sample size $(n=8)$. Nevertheless, the average contribution of the paretic leg amounted to as little as $10-20 \%$ while bearing $40-45 \%$ of the body weight, whereas in healthy subjects the weight borne on a leg equaled its contribution to postural control when adopting an asymmetric position [29]. 
The study by Mansfield and coworkers [10] provided further insight into the temporal aspects of corrective actions under the paretic and nonparetic legs as assessed by crosscorrelation of the individual COP trajectories. Greater WBA was associated with poorer synchronization (i.e., lower crosscorrelation coefficients) of COP trajectories $\left(R^{2}=0.19-0.23\right)$ [10], which indicated that the paretic and nonparetic legs less adequately worked together in controlling balance when adopting a more asymmetric weight distribution. It was shown that the synchronization of left and right COP trajectories was almost perfect (i.e., cross-correlation coefficients close to 1.0) in healthy persons, but it was not investigated whether and how this would be affected by WBA.

The effect of WBA on the timing of balance correcting responses after support-surface translations was investigated by Marigold et al. [26]. This was the only study using a withinsubjects design. In contrast to healthy controls, people with stroke demonstrated largely absent modulation of response latencies and amplitudes with different degrees of WBA. In other words, imposing weight-bearing symmetry resulted in only minimal improvement in the corrective postural responses of the paretic leg. Overall, the muscular responses of the patients were delayed compared to the healthy controls. These findings suggest that WBA is not the primary cause of the reduced postural stability after stroke. This suggestion is supported by several other observations. First, the degree of postural instability after stroke greatly exceeds the effect of adopting different degrees of WBA on postural stability in healthy persons (within the ranges that are commonly observed after stroke) [11,33]. Second, the disproportionately low contribution of the paretic leg to postural control (relative to the amount of weight borne on the paretic leg) as reported by van Asseldonk et al. [29] also argues against a major role for WBA in the causation of postural instability after stroke.

Alternatively, we suggest that WBA after stroke may be regarded as a compensatory strategy to enhance the kinetic contribution of the nonparetic leg to balance. Several findings support this notion. First, it has been found in a longitudinal cohort study that, although WBA decreased within the first weeks after stroke and postural stability improved over a period of at least another two months, the regulatory activity of the paretic leg (expressed as COP velocity) did not improve [11]. In the same vein, the contribution of the paretic leg to postural control was found to be as little as $10-20 \%$ despite good recovery of ambulatory capacity (FAC 4-5) and balance performance (BBS > 45) in the chronic phase after stroke [29]. These findings suggest that improvement of postural stability after stroke is primarily driven by compensatory strategies rather than by the restoration of motor control of the affected leg. Second, Roerdink and coworkers found that both WBA and a reduced contribution of the paretic leg to postural control were most evident in individuals with a lack of selective muscle control of the paretic leg [24]. These patients may have used WBA to enhance the kinetic contribution of the nonparetic leg to balance.

Compensation for decreased regulatory activity by the paretic leg as a possible explanation for persistent WBA after stroke does, however, not preclude the possibility of other underlying mechanisms. For instance, recent evidence suggests that misperception of the postural and visual vertical after stroke may also contribute to WBA while standing, particularly in patients with visuospatial hemineglect $[9,34]$. From this perspective, WBA may be regarded as a primary impairment rather than a secondary compensation.

Although WBA towards the nonparetic leg may be a beneficial compensation for the reduced regulatory activity by the paretic leg while quiet standing, it remains to be investigated to what extent WBA is advantageous for dynamic postural stability, such as when stepping to recover balance after an external perturbation. In a recent study it was found that after stroke WBA towards the nonaffected side was associated with an increased likelihood of stepping with the paretic leg in response to a forward perturbation [35]. It was suggested that this strategy may be less effective to restore balance than to step with the nonparetic leg. Further research should, therefore, shed light on the effects of different degrees of WBA on a variety of postural tasks.

4.2. Clinical Balance Performance. Whereas static and dynamic posturography provide information on underlying postural control mechanisms, clinical balance tests such as the Berg Balance Scale and the Functional Reach test evaluate functional capacities. They allow for adaptive strategies to compensate for the underlying impairments to accomplish the required task. The results of the two studies that reported on the correlation between WBA and clinical balance tests do not provide conclusive evidence for the magnitude or direction of a possible association. First, the association between greater WBA and lower BBS scores was indirectly derived from a larger regression model as reported in the study by Mansfield et al. [30], which may explain the relatively small explained variance. As this study was not originally designed to investigate this association and the regression model contained other measures of postural control as well, it is difficult to draw any conclusions from this finding.

The study by Pereira et al. (2010) was the only one that reported greater WBA to be associated with better postural stability as measured with the Functional Reach test, but this only concerned the individuals bearing more weight on the nonparetic leg [27]. This finding suggests that WBA may be an effective compensatory strategy during functional (reach) tasks. Yet, further research is needed to confirm this notion.

4.3. Falls in Daily Life. The ultimate consequence of postural instability is falling, which was therefore regarded as a measure of postural stability as well. Although the two studies that reported on the association between WBA and falls in daily life found no significant correlations, these results should be interpreted cautiously. First, the study by Mansfield et al. was not designed to specifically assess this association [30]. Second, in the study by Sackley, the fall data collection did not comply to the currently accepted methods, which may have influenced the results [8].

4.4. Limitations. The number of studies reporting on the association between WBA and postural stability was limited $(N=9)$, with only seven studies being originally designed 
to investigate this association. Another limitation was the variety of balance measures that assessed different aspects of postural control, which limited the possibility to compare outcomes. This problem was only partially overcome by categorization of the balance measures. Besides differences in measures of postural stability, study populations varied greatly in terms of poststroke duration and functional capacities. Although most studies only considered participants with unilateral stroke, two studies also included patients with bilateral stroke $[8,30]$. Furthermore, two studies $[24,30]$ did not consider the direction of WBA (towards the paretic or nonparetic side), while the study by Pereira et al. [27] demonstrated that associations between WBA and postural stability may be dependent on this direction.

The reporting of confounding bias was poor in all studies except one [30]. In only three studies a method to correct for confounders was applied $[24,26,30]$. One important confounder that was often not accounted for was stroke severity. Postural instability and WBA are probably both related to stroke severity $[7,9,11,34,36,37]$ and, consequently, these phenomena will be strongly correlated. Yet, to better understand the causal relationship between WBA and postural stability after stroke, the effect of different degrees of imposed WBA on postural control should be investigated within the same group of patients.

\section{Conclusion}

Overall, the studies included in this review suggest that WBA after stroke is associated with increased postural sway as well as with poorer between-limb synchronization of COP trajectories. These associations were obtained from cross-sectional studies, which do not provide insight into causal relationships. Yet, the one study that imposed different degrees of weight-bearing symmetry does not provide support for WBA being a main cause of postural instability in people with stroke. Whether adopting an asymmetric weight distribution (in favour of the nonparetic leg) is detrimental or beneficial for both static and dynamic postural stability after stroke remains to be investigated for various degrees of stroke severity (in particular the severity of paresis).

\section{Appendix}

\section{A. Search Strategy MEDLINE}

(1) $(((((($ “'Stroke”[Mesh]) OR “Brain Infarction”[Mesh]) OR “Cerebrovascular Disorders" [Mesh]) OR "Brain Ischemia" [Mesh]) OR "Paresis"[Mesh]) OR "Hemiplegia”[Mesh]) OR "Intracranial Hemorrhages"[Mesh]) OR “Intracranial Embolism and Thrombosis"[Mesh]

(2) "cva"

(3) "cerebral"

(4) "stroke"

(5) "cerebrovascular"

(6) $1,2,3,4,5$
(7) ("Postural Balance"[Mesh]) OR "Posture"[Mesh]

(8) "balance/physiology"

(9) "balance/equilibrium"

(10) "balance/coordination"

(11) "balance"

(12) "balance control"

(13) "posture"

(14) "postural"

(15) $7,8,9,10,11,12,13,14$

(16) “weight-bearing”[Mesh]

(17) "weight bearing"

(18) "weight bearing/physiology"

(19) "weight bearing asymmetry"

(20) $16,17,18,19$

(21) 6, AND 15, AND 20.

\section{Authors' Contribution}

Both the first and the second authors equally contributed to the paper.

\section{References}

[1] B. Kollen, G. Kwakkel, and E. Lindeman, "Functional recovery after stroke: a review of current developments in stroke rehabilitation research," Reviews on Recent Clinical Trials, vol. 1, no. 1, pp. 75-80, 2006.

[2] I. G. van de Port, G. Kwakkel, V. P. Schepers, and E. Lindeman, "Predicting mobility outcome one year after stroke: A Prospective Cohort Study," Journal of Rehabilitation Medicine, vol. 38, no. 4, pp. 218-223, 2006.

[3] B. J. Kollen, G. Kwakkel, and E. Lindeman, "Longitudinal robustness of variables predicting independent gait following severe middle cerebral artery stroke: A Prospective Cohort Study," Clinical Rehabilitation, vol. 20, no. 3, pp. 262-268, 2006.

[4] V. Weerdesteyn, M. de Niet, H. J. R. van Duijnhoven, and A. C. H. Geurts, "Falls in individuals with stroke," Journal of Rehabilitation Research \& Development, vol. 45, no. 8, pp. 1195-1213, 2008.

[5] S. F. Mackintosh, K. D. Hill, K. J. Dodd, P. A. Goldie, and E. G. Culham, "Balance score and a history of falls in hospital predict recurrent falls in the 6 months following stroke rehabilitation," Archives of Physical Medicine and Rehabilitation, vol. 87, no. 12, pp. 1583-1589, 2006.

[6] A. S. Aruin, T. Hanke, G. Chaudhuri, R. Harvey, and R. Noel, "Compelled weightbearing in persons with hemiparesis following stroke: the effect of a lift insert and goal-directed balance exercise," Journal of Rehabilitation Research and Development, vol. 37, no. 1, pp. 65-72, 2000.

[7] J. Mizrahi, P. Solzi, H. Ring, and R. Nisell, "Postural stability in stroke patients: vectorial expression of asymmetry, sway activity and relative sequence of reactive forces," Medical and Biological Engineering and Computing, vol. 27, no. 2, pp. 181-190, 1989.

[8] C. M. Sackley, "Falls, sway, and symmetry of weight-bearing after stroke," International Disability Studies, vol. 13, no. 1, pp. $1-4,1991$. 
[9] J. Barra, L. Oujamaa, V. Chauvineau, P. Rougier, and D. Pérennou, "Asymmetric standing posture after stroke is related to a biased egocentric coordinate system," Neurology, vol. 72, no. 18, pp. 1582-1587, 2009.

[10] A. Mansfield, C. J. Danells, E. Inness, G. Mochizuki, and W. E. McIlroy, "Between-limb synchronization for control of standing balance in individuals with stroke," Clinical Biomechanics, vol. 26, no. 3, pp. 312-317, 2011.

[11] M. De Haart, A. C. Geurts, S. C. Huidekoper, L. Fasotti, and J. Van Limbeek, "Recovery of standing balance in postacute stroke patients: A Rehabilitation Cohort Study," Archives of Physical Medicine and Rehabilitation, vol. 85, no. 6, pp. 886-895, 2004.

[12] B. J. Kollen, S. Lennon, B. Lyons et al., "The effectiveness of the bobath concept in stroke rehabilitation what is the evidence?" Stroke, vol. 40, no. 4, pp. e89-e97, 2009.

[13] P. Davies, Steps to Follow: The Comprehensive Treatment of Patients with Hemiplegia, Springer, 2nd edition, 2000.

[14] S. Chaudhuri and A. S. Aruin, "The effect of shoe lifts on static and dynamic postural control in individuals with hemiparesis," Archives of Physical Medicine and Rehabilitation, vol. 81, no. 11, pp. 1498-1503, 2000.

[15] C. H. Chen, K. H. Lin, T. W. Lu et al., "Immediate effect of lateral-wedged insole on stance and ambulation after stroke," American Journal of Physical Medicine and Rehabilitation, vol. 89, no. 1, pp. 48-55, 2010.

[16] M. C. Dault, M. de Haart, A. C. H. Geurts, I. M. P. Arts, and B. Nienhuis, "Effects of visual center of pressure feedback on postural control in young and elderly healthy adults and in stroke patients," Human Movement Science, vol. 22, no. 3, pp. 221-236, 2003.

[17] R. P. S. Van Peppen, M. Kortsmit, E. Lindeman, and G. Kwakkel, "Effects of visual feedback therapy on postural control in bilateral standing after stroke: a systematic review," Journal of Rehabilitation Medicine, vol. 38, no. 1, pp. 3-9, 2006.

[18] A. S. Pollock, B. R. Durward, P. J. Rowe et al., "What is balance?" Clinical Rehabilitation, vol. 14, no. 4, pp. 402-406, 2000.

[19] D. Moher, A. Liberati, J. Tetzlaff, and D. G. Altman, "Preferred reporting items for systematic reviews and meta-analyses: The PRISMA statement," British Medical Journal, vol. 339, no. 7716, pp. 332-336, 2009.

[20] S. Sanderson, I. D. Tatt, and J. P. T. Higgins, "Tools for assessing quality and susceptibility to bias in observational studies in epidemiology: a systematic review and annotated bibliography," International Journal of Epidemiology, vol. 36, no. 3, pp. 666676, 2007.

[21] E. von Elm, D. G. Altman, M. Egger, S. J. Pocock, P. C. Gøtzsche, and J. P. Vandenbroucke, "The Strengthening the Reporting of Observational Studies in Epidemiology (STROBE) statement: guidelines for reporting observational studies," The Lancet, vol. 370, no. 9596, pp. 1453-1457, 2007.

[22] J. P. Vandenbroucke, E. Von Elm, D. G. Altman et al., "Strengthening the Reporting of Observational Studies in Epidemiology (STROBE): explanation and elaboration," Epidemiology, vol. 18, no. 6, pp. 805-835, 2007.

[23] R. H. H. Groenwold, A. M. M. Van Deursen, A. W. Hoes, and E. Hak, "Poor Quality of Reporting Confounding Bias in Observational Intervention Studies: a systematic review," Annals of Epidemiology, vol. 18, no. 10, pp. 746-751, 2008.

[24] M. Roerdink, A. C. H. Geurts, M. De Haart, and P. J. Beek, “On the relative contribution of the paretic leg to the control of posture after stroke," Neurorehabilitation and Neural Repair, vol. 23, no. 3, pp. 267-274, 2009.
[25] D. S. Marigold and J. J. Eng, "The relationship of asymmetric weight-bearing with postural sway and visual reliance in stroke," Gait and Posture, vol. 23, no. 2, pp. 249-255, 2006.

[26] D. S. Marigold, J. J. Eng, and I. J. Timothy, "Modulation of ankle muscle postural reflexes in stroke: influence of weight-bearing load," Clinical Neurophysiology, vol. 115, no. 12, pp. 2789-2797, 2004.

[27] L. C. Pereira, A. C. Botelho, and E. F. Martins, "Relationships between body symmetry during weight-bearing and functional reach among chronic hemiparetic patients," Revista Brasileira de Fisioterapia, vol. 14, no. 3, pp. 259-266, 2010.

[28] S. H. Peurala, P. Könönen, K. Pitkänen, J. Sivenius, and I. M. Tarkka, "Postural instability in patients with chronic stroke," Restorative Neurology and Neuroscience, vol. 25, no. 2, pp. 101108, 2007.

[29] E. H. F. van Asseldonk, J. H. Buurke, B. R. Bloem et al., "Disentangling the contribution of the paretic and non-paretic ankle to balance control in stroke patients," Experimental Neurology, vol. 201, no. 2, pp. 441-451, 2006.

[30] A. Mansfield, G. Mochizuki, E. L. Inness, and W. E. McIlroy, "Clinical correlates of between-limb synchronization of standing balance control and falls during inpatient stroke rehabilitation," Neurorehabilitation and Neural Repair, vol. 26, no. 6, pp. 627-635, 2012.

[31] A. C. H. Geurts, B. Nienhuis, and T. W. Mulder, "Intrasubject variability of selected force-platform parameters in the quantification of postural control," Archives of Physical Medicine and Rehabilitation, vol. 74, no. 11, pp. 1144-1150, 1993.

[32] D. Lafond, H. Corriveau, R. Hébert, and F. Prince, "Intrasession reliability of center of pressure measures of postural steadiness in healthy elderly people," Archives of Physical Medicine and Rehabilitation, vol. 85, no. 6, pp. 896-901, 2004.

[33] L. C. Anker, V. Weerdesteyn, I. J. W. van Nes, B. Nienhuis, H. Straatman, and A. C. H. Geurts, "The relation between postural stability and weight distribution in healthy subjects," Gait and Posture, vol. 27, no. 3, pp. 471-477, 2008.

[34] D. Pérennou, "Postural disorders and spatial neglect in stroke patients: a strong association," Restorative Neurology and Neuroscience, vol. 24, no. 4-6, pp. 319-334, 2006.

[35] A. Mansfield, E. L. Inness, B. Lakhani, and W. E. McIlroy, "Determinants of limb preference for initiating compensatory stepping poststroke," Archives of Physical Medicine and Rehabilitation, vol. 93, no. 7, pp. 1179-1184, 2012.

[36] N. Genthon, A. S. Gissot, J. Froger, P. Rougier, and D. Pérennou, "Posturography in patients with stroke: estimating the percentage of body weight on each foot from a single force platform," Stroke, vol. 39, no. 2, pp. 489-491, 2008.

[37] N. Genthon, P. Rougier, A. S. Gissot, J. Féroger, J. Pélissier, and D. Pérennou, "Contribution of each lower limb to upright standing in stroke patients," Stroke, vol. 39, no. 6, pp. 1793-1799, 2008 . 


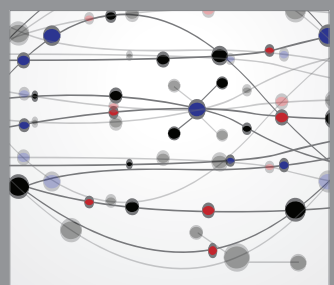

The Scientific World Journal
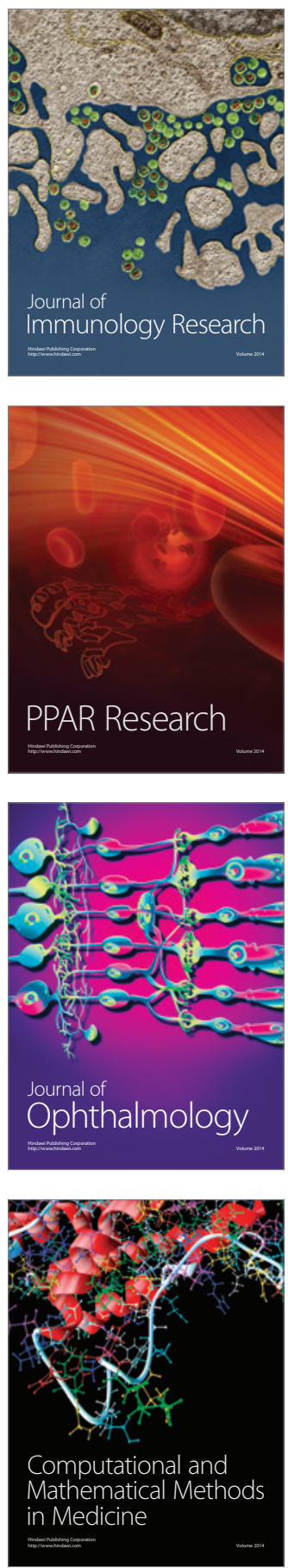

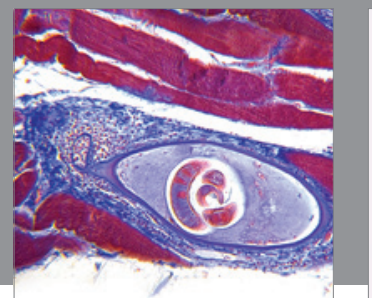

Gastroenterology

Research and Practice
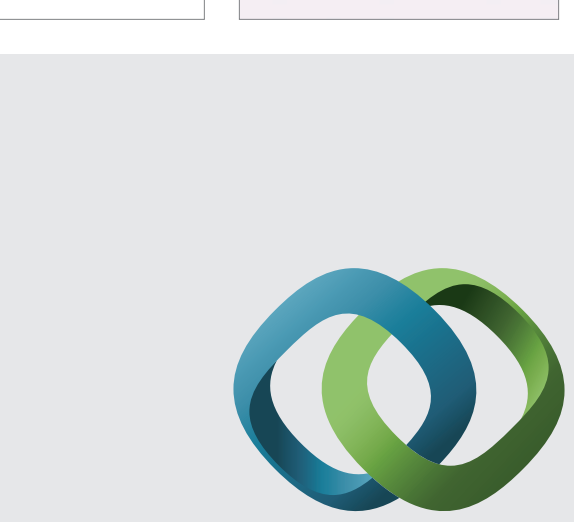

\section{Hindawi}

Submit your manuscripts at

http://www.hindawi.com
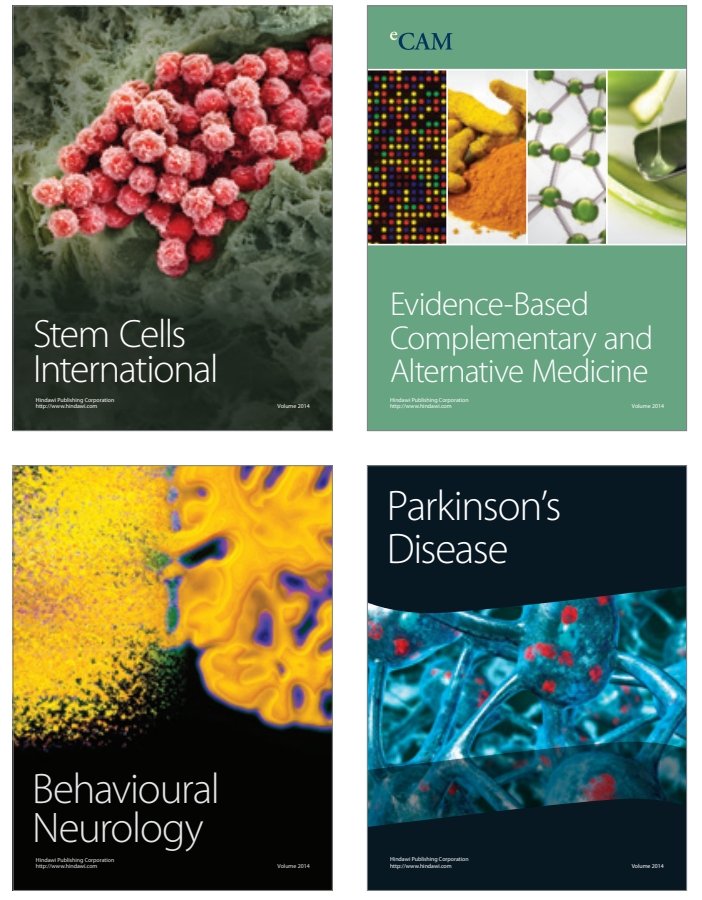
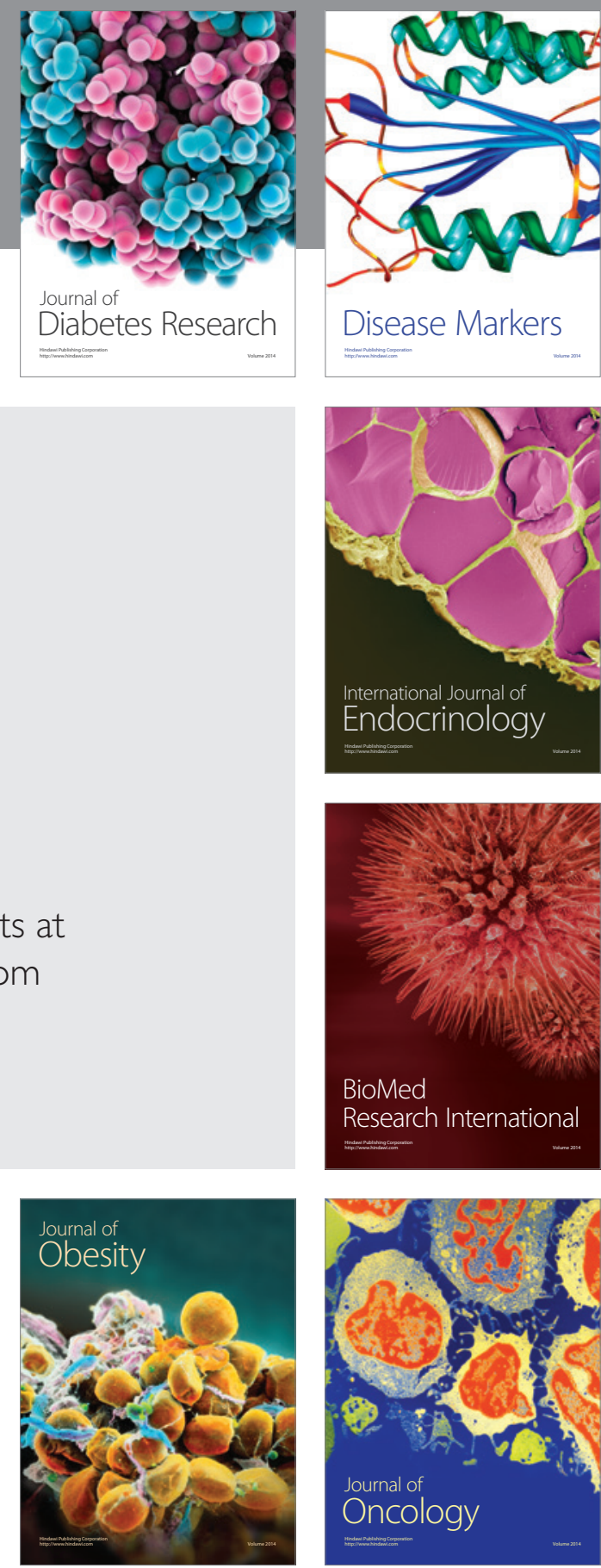

Disease Markers
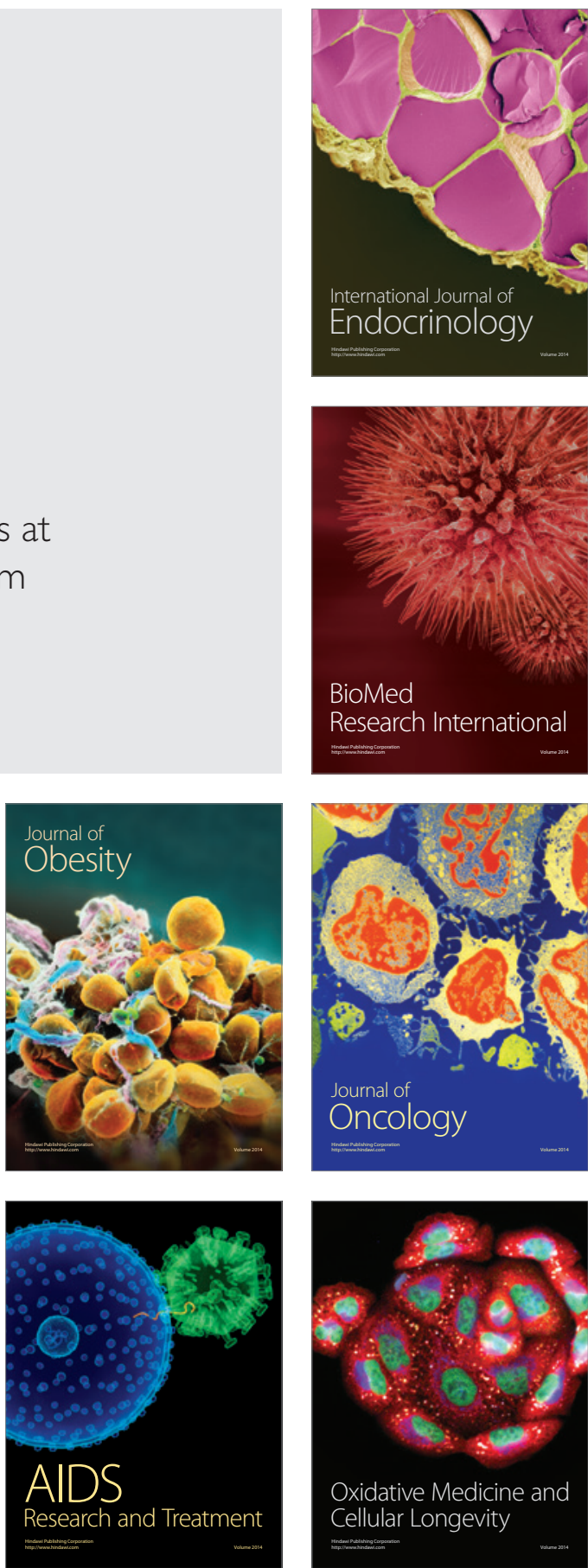